\title{
The cyclic GMP/protein kinase G pathway as a therapeutic target in head and neck squamous cell carcinoma
}

\author{
Traci R Tuttle ${ }^{\mathrm{a}}$, Michelle L Mierzwa ${ }^{\mathrm{b}}$, Susanne I Wells ${ }^{\mathrm{c}}$, Sejal R Fox ${ }^{\mathrm{a}}$, Nira Ben-Jonathan ${ }^{\mathrm{a} *}$ \\ ${ }^{a}$ Department of Cancer Biology, University of Cincinnati School of Medicine, Cincinnati, OH \\ 45267, USA \\ ${ }^{b}$ Department of Radiation Oncology, University of Cincinnati School of Medicine, Cincinnati \\ OH 45267, USA
}

' Division of Oncology, Cancer and Blood Diseases Institute, Children's Hospital Medical Center, Cincinnati, OH, 45229, USA

"Corresponding author at: Department of Cancer Biology, The University of Cincinnati, 3125 Eden Ave, Cincinnati, OH 45267, USA

Key Words: cGMP, PKG, sGC, PDE5, HNSCC, Tadalafil

\begin{abstract}
Head and neck squamous cell carcinoma (HNSCC) is an aggressive disease with high mortality. Treatments, which can result in significant morbidity, have not substantially changed in three decades. The second messenger cyclic GMP (cGMP), which targets protein kinase G (PKG), is generated by guanylate cyclases (GCs), and is rapidly hydrolyzed by phosphodiesterases (PDEs). Activation of the cGMP/PKG pathway is antineoplastic in several cancer types, but its impact on HNSCC has not been fully exploited. We found differential expression of critical components of this pathway in four HNSCC cell lines. Several activators of soluble GC (sGC) as well as
\end{abstract}


inhibitors of PDE5, increased intracellular cGMP, reduced cell viability, and induced apoptosis in HNSCC cells. The apoptotic effects of the sGC activator BAY 41-2272 and the PDE5 inhibitor Tadalafil (Cialis) were mediated by PKG. Furthermore, Tadalafil substantially reduced the growth of CAL27-derived tumors in athymic mice. Several drugs which either activate sGC or inhibit PDE5 are approved for treatment of nonmalignant conditions. These drugs could be repurposed as novel and effective therapeutics in patients with head and neck cancer.

\section{Introduction}

Head and neck cancer is the $6^{\text {th }}$ most common cancer, with $\sim 600,000$ new cases diagnosed annually worldwide [1]. These tumors frequently occur in the oral cavity, oropharynx, hypopharynx and larynx, and $>90 \%$ are histologically classified as squamous cell carcinomas (HNSCC). Risk factors have historically included tobacco use and heavy alcohol consumption. Human papilloma virus (HPV)-positive tumors are increasing in prevalence and in general have improved prognosis [2]. Yet, the overall five year survival rate for HNSCC is less than 50\% [3]. Treatments include surgery, radiotherapy, chemotherapy or their combinations. Disease progression as well as treatment can result in adverse side effects such as facial disfigurement, difficulty swallowing and speech impediment, which severely reduce the quality of life [4]. Hence, more effective therapeutic approaches for HNSCC are urgently needed.

The cyclic GMP (cGMP)/protein Kinase G (PKG) cascade is recognized as an endogenous apoptotic pathway in a number of cancer types, particularly breast and colon [5-10]. Cyclic GMP is generated from GTP by two distinct guanylate cyclases (GCs) types. Particulate GCs (pGCs) are transmembrane receptors for natriuretic peptides, while soluble GC (sGC) is a cytosolic 
enzyme $[11,12]$, that serves as a receptor for nitric oxide (NO), a cell permeable second messenger. Once produced, cGMP can be rapidly hydrolyzed by cyclic nucleotide phosphodiesterases (PDEs), a superfamily of 12 members that differ in structure, catalytic properties, and subcellular localization [13]. The PDEs are grouped into three classes: PDE4, 7 and 8 that hydrolyze cAMP, PDE5, 6 , and 9 that breakdown cGMP, and the remainder have dual activity. The main downstream effectors of cGMP are two serine-threonine kinases, PKG-I and PKG-II, which share a number of targets with protein kinase A (PKA), while others are distinct [14].

Several drugs which increase intracellular cGMP are approved for treatment of nonmalignant conditions. For example, selective PDE5 inhibitors such as Tadalafil (Cialis), Sildenafil (Viagra) and Vardenafil (Levitra) are used to treat erectile dysfunction [15]. Sodium nitroprusside (SNP), a nitric oxide donor, is used as an acute vasodilator [16], while Riociguat, an NO-independent sGC stimulator, is approved for the treatment of pulmonary hypertension [17]. There is significant potential for repurposing these drugs as antineoplastic agents in many cancers.

The objectives of this study were to: a) examine expression of components of the sGC/PDE/PKG signaling cascade, and b) determine the effects of cGMP pathway activators on the growth and survival of HNSCC cells. We are reporting that activation of the sGC/cGMP/PKG cascade by sGC stimulators and/or by PDE5 inhibitors markedly increase apoptosis in cultured HNSCC cells. Furthermore, administration of Tadalafil to athymic mice with HNSCC xenografts suppresses tumor growth. Figure 1A illustrates critical components of the cGMP/PKG apoptotic pathway and its augmentation by diverse drugs. 


\section{Materials and Methods}

\subsection{Cell lines and culture}

UMSCC-1 (UM1; oral cavity, HPV-), UMSCC-6 (UM6; oropharynx, HPV-), UMSCC-47 (UM47; tongue, HPV+) cells were purchased from the University of Michigan, while CAL27 (tongue, HPV-) cells were from the American Type Culture Collection (ATCC). Cells were maintained in DMEM containing 10\% FBS (Atlanta Biologicals, Flowery Branch, GA) and 50 $\mu \mathrm{g} / \mathrm{ml}$ normocin (Invivogen, San Diego, CA). For experiments, cells were starved for $24 \mathrm{~h}$ and then incubated with various treatments in DMEM containing 2\% charcoal/dextran-stripped FBS (Atlanta Biologicals) unless otherwise noted.

\subsection{Drugs and inhibitors}

The NO donors S-Nitroso-N-Acetyl-D,L-Penicillamine (SNAP) and (Z)-1-[N-(2-aminoethyl)-N(2-ammonioethyl)amino]diazen-1-ium-1,2-diolate (DETA NONOate), were obtained from Cayman Chemical (Ann Arbor, MI), and sodium nitroprusside (SNP) was obtained from Sigma Aldrich (St. Louis, MO). The sGC stimulators BAY 41-2272 (BAY) and YC-1, and the sGC inhibitor 1H-[1,2,4] oxadiazolo[4,3-a]quinoxalin-1-one (ODQ) were from Cayman. The PDE5 inhibitors Tadalafil (Cialis) and Sildenafil (Viagra) were from Selleck Chemical (Houston, TX). The PKG inhibitor KT5823 was from Cayman.

\subsection{Conventional PCR}

RNA was isolated using RNAspin Mini kit (GE Healthcare, Milwaukee WI), and reversed transcribed using a first strand RT kit from Qiagen (Germantown, MD). PCR amplification was done with primers for cGMP pathway components or $\beta 2$-Microglobulin $(B 2 M)$, used as a reference gene (Table I). PCR was done using DreamTaq Green PCR Mastermix (Life Technologies, Carlsbad, CA) in an Eppendorf Master Cycler (Westbury, NY). Cycle conditions 
were: $95 \mathrm{C}$ for $3 \mathrm{~min}$, followed by 35 cycles of $95 \mathrm{C}, 58 \mathrm{C}$, and $72 \mathrm{C}$, each for $30 \mathrm{sec}$. Products were resolved on $1.5 \%$ agarose gel with ethidium bromide and digitally imaged.

\section{4 cGMP analysis}

Cells were plated at 250,000 cells/well in 12-well plates, and treated with various compounds for 60 min. After lysis in $0.1 \mathrm{M} \mathrm{HCl}$ and centrifugation, cGMP in supernatants was quantified by a colorimetric competitive ELISA kit from Cayman.

\subsection{Cell viability and BrdU incorporation assays}

Cells were plated at 3000 cells/well in 96-well plates and were treated for $72 \mathrm{~h}$. Cell viability was determined by the MTT assay (Affymetrix, Cleveland, OH). For the BrdU DNA synthesis assay, cells were treated for $24 \mathrm{~h}$ and cell lysates were analyzed using an ELISA kit (Millipore, San Diego, CA).

\subsection{Flow Cytometry}

Cells were plated at 300,000 cells/well in 6-well plates. For inhibitor studies, ODQ or KT5823 was added $1 \mathrm{~h}$ before treatment with other drugs. After $72 \mathrm{~h}$, apoptosis was determined using FITC/Annexin V Apoptosis Detection Kit (BD Biosciences, San Jose, CA). Briefly, cells were incubated with Annexin $\mathrm{V}$ and propidium iodide for $15 \mathrm{~m}$ and then analyzed by flow cytometry, using a Cell Lab Quanta SC Flow Cytometer with accompanying software (Beckman Coulter). About 10,000 gated events were collected per treatment.

\subsection{Western blotting}

Cells were treated for $24 \mathrm{~h}$ and then lysed on ice in RIPA buffer containing a cocktail of protease inhibitors (Sigma). Protein concentrations were determined by a BCA protein assay kit (Life Technologies), and $40 \mu \mathrm{g}$ were resolved on $12 \%$ SDS-PAGE, and transferred to PVDF 
membranes. After blocking with $0.5 \%$ BSA, membranes were incubated in primary antibodies against Caspase-9 (Cell Signaling Technologies, Danvers, MA) for $24 \mathrm{~h}$, followed by several washes and incubation with HRP-conjugated secondary antibody (GE Healthcare). Blots were developed using SuperSignal West Pico chemiluminescence substrate (Life Technologies). Membranes were re-probed with $\beta$-actin antibody (Sigma) as a loading control.

\subsection{Clonogenic assay}

Cells were plated in growth media at 150 cells/well in 6-well plates, allowed to attach for $6 \mathrm{~h}$ and then treated for $72 \mathrm{~h}$. Media were replaced, and the cells were maintained in growth media for two weeks. Colonies were fixed with glutaraldehyde $(6.0 \% \mathrm{v} / \mathrm{v})$, stained with crystal violet $(0.5 \% \mathrm{w} / \mathrm{v})$ and photographed. Colonies containing $\geq 50$ cells were counted.

\subsection{Animals and xenograft studies}

Eight-week old female athymic $n u / n u$ mice were obtained from Charles River Laboratories (Wilmington, MA). Mice were housed 4/cage under sterile conditions, and acclimated for 7-10 days before experiments. The animal use protocol (\#04-06-29-01) was approved by the University of Cincinnati Institutional Animal Care \& Use Committee. CAL27 cells were suspended 1:1 in PBS/Matrigel (BD Biosciences, Franklin Lakes, NJ) and $2 \times 10^{6}$ cells/100 $\mu 1$ were inoculated sc into the flank. Tumor dimensions were measured twice/week, and volume was calculated as length $\mathrm{x}$ width ${ }^{2} \mathrm{x} 0.52$. When tumors reached $\sim 200 \mathrm{~mm}^{3}$, Alzet osmotic minipumps (model 1004, Durect Corporation; Cupertino, CA) with a $100 \mu 1$ reservoir and rated for a continuous delivery at $0.09 \mu \mathrm{l} / \mathrm{hr}$ for 4 weeks, were implanted sc. The pumps delivered vehicle (polyethylene glycol 400; Sigma) or Tadalafil $(1 \mathrm{mg} / \mathrm{kg} /$ day). Each treatment included 12 mice. Tumor volumes were measured for an additional 3 weeks, at which time mice were euthanized and tumors were removed and weighed. 


\subsection{Statistics}

Student's t-test or ANOVA were used where appropriate. P-values $\leq 0.05$ were considered significant. All experiments were repeated at 2-3 times, unless otherwise noted.

\section{Results}

\subsection{HNSCC cell lines express essential components of the $s G C / P D E / P K G$ signaling cascade}

The sGC enzyme is a heterodimer comprised of $\alpha$ subunits: sGC $\alpha 1$ or sGC $\alpha 2$ (encoded by GUCY1A3 or GUCY1A2, respectively), and a $\beta$ subunit: sGC $\beta 1$ (encoded by GUCY1B3). As determined by conventional PCR, all four HNSCC cell lines expressed the common $\beta$ subunit and GUCY1A3, while only UM47 cells expressed detectable levels of GUCY1A2 (Fig. 1B). Since in addition to inhibiting PDE5, Tadalafil can also inhibit PDE11 [18], we examined the expression of both genes. All cell lines expressed PDE5A, while only CAL27 cells expressed appreciable levels of PDE11A. Two PKG genes encoding PKG-I (PRKG1) and PKG-II

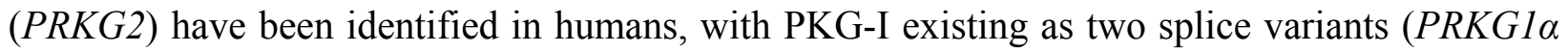
and $P R K G 1 \beta)$. All four cell lines expressed $P R K G 2$ and $P K G 1 \beta$, while none expressed detectable levels of $P K G 1 \alpha$ (Fig. 1B).

\subsection{Tadalafil, BAY 41-2272 and SNP increase cGMP in HNSCC cells}

We next examined whether drugs which stimulate sGC or inhibit PDE5 alter cGMP levels in HNSCC cells. UM47 and CAL27 cells were treated for 60 minutes with the sGC activator BAY 41-2272 (BAY; $10 \mu \mathrm{M}$ ), the NO donor sodium nitroprusside (SNP; 1mM), or the PDE5 inhibitor Tadalafil (Tad; $50 \mu \mathrm{M}$ ), or with a combination of BAY and Tadalafil or SNP and Tadalafil. As determined by an ELISA, all three drugs significantly increased cGMP content, with BAY being most effective in both cell lines (Fig. 1C); UM47 cells were considerably more responsive than 
CAL27 cells. As expected, combined treatments were more effective than treatment with any single drug. Similar results were obtained with UM1 and UM6 cells (data not shown).

\subsection{Various sGC stimulators and PDE5 inhibitors decrease the viability of HNSCC cells}

The effects of sGC stimulators and PDE5 inhibitors on cell viability were compared among the four HNSCC cell lines. As determined by MTT assay, cell viability was decreased by the NO donors DETA-NONOate, SNAP and SNP (Fig. 2A-C). Treatment with the NO-independent sGC stimulators BAY (Fig. 2D) and YC-1 (Fig. 2E) also decreased the viability of the four cell lines in a dose-dependent manner. BAY was effective at much lower doses than YC-1. As illustrated in Fig. 2F and Fig. 2G, both PDE5 inhibitors Tadalafil and Sildenafil decreased cell viability, with the more stable Tadalafil having a more pronounced effect. Co-treatment with BAY and Tadalafil was more effective than either drug alone (Fig. 2H).

\subsection{BAY 41-2272 and Tadalafil reduce cell proliferation and induce apoptosis}

Since decreased cell viability can reflect a suppression of cell proliferation and/or an increased cell death, we next examined the effects of BAY and Tadalafil on both parameters. As determined by BrdU incorporation, a 24-hr treatment with $10 \mu \mathrm{M}$ BAY modestly, but significantly, decreased the proliferation of all four cell lines (Fig. 3A). Tadalafil at $50 \mu \mathrm{M}$ decreased the proliferation of UM47 and CAL27, but not of UM1 or UM6 cells. As determined by Annexin V staining and flow cytometry, both BAY and Tadalafil substantially increased apoptosis in all four cell lines (Fig. 3B). Both drugs also induced Caspase-9 cleavage in CAL27 and UM47 cells (Fig. 3C), confirming an induction of apoptosis. Collectively, these data indicate that the cGMP activating drugs exert both cytotoxic and cytostatic effects in HNSCC cells. 


\subsection{BAY and Tadalafil decrease clonogenic survival of HNSCC cells}

Clonogenic survival assays are commonly employed to determine whether cancer cells recover over time from the effects of cytotoxic treatments [19]. CAL27, UM6 and UM47 cells were plated at very low density and Tadalafil $(50 \mu \mathrm{M})$ or BAY $(10 \mu \mathrm{M})$ were added $4-6 \mathrm{~h}$ later for 72 h. The cells were then maintained in growth media for two weeks in the absence of drugs. Fig. 4A-C show that both drugs sharply reduced the number of surviving colonies in all three cell lines, with BAY being more effective than Tadalafil.

3.6 Inhibition of sGC or PKG reverses the apoptotic effects of BAY and Tadalafil in UM47 cells

We next used selective enzyme inhibitors to examine whether sGC or PKG mediate the induction of apoptosis by BAY or Tadalafil in UM47 cells. As determined by Annexin/PI flow cytometry, the PKG inhibitor KT5823 reversed the apoptotic effects of both BAY and Tadalafil (Fig. 5A and 5B). Inhibition of sGC with ODQ reversed the effects of BAY, but not of Tadalafil. As evident in Fig 5B, within $72 \mathrm{~h}$ of treatment, BAY and Tadalafil effectively increase the number of cells in early and late apoptosis.

\subsection{Tadalafil strongly inhibits the growth of CAL27 xenografts}

A mouse xenograft model was used to examine the effect of Tadalafil on CAL27 tumor growth in vivo. After tumors reached a moderate size, Alzet osmotic mini-pumps delivering Tadalafil $(1 \mathrm{mg} / \mathrm{kg} / \mathrm{day})$ or vehicle were implanted sc. Treatment with Tadalafil resulted in a $60 \%$ reduction in tumor volume (Fig. 6A) and a 70\% decrease in tumor weight (Fig. 6B). There were no observed changes in weight or behavior of the treated mice. Photographs of mice treated with Tadalafil or vehicle and their excised tumors are presented in Fig 6C. 


\section{Discussion}

This is the first report on the expression and functions of the cGMP/PKG pathway in head and neck cancer. Activation of this pathway in four HNSCC cell lines, derived from different anatomical origins and genetic backgrounds, suppressed cell viability, reduced cell proliferation, and induced apoptosis. Tadalafil, a long acting PDE5 inhibitor, markedly suppressed the growth of HNSCC xenografts in vivo.

Activation of sGC by the NO donor SNP, as well as by the direct sGC stimulators BAY and YC1, increased cGMP accumulation and decreased cell viability. Our data indicate that the druginduced suppression of cell viability reflects the combined effect of reduced cell proliferation and increased apoptosis. These findings are supported by the reports that NO donors and direct sGC stimulators exerted anti-tumorigenic effects via the sGC/cGMP signaling pathway in ovarian, breast and prostate cancers [5, 7]. On the other hand, anti-apoptotic actions of sGC/cGMP pathway in lung and ovarian cancer $[20,21]$ have also been reported, suggesting that the ultimate outcome of cGC activation depends on cell-specific downstream effectors.

The intracellular levels of cGMP are determined by a dynamic balance between its synthesis by GCs and hydrolysis by PDEs. We found robust expression of PDE5 in all HNSCC cell lines. Treatment of these cells with Tadalafil increased cGMP, reduced cell viability and induced apoptosis. The involvement of PKG was established by the reversal of the Tadalafil-induced apoptosis by the PKG inhibitor KT5823. Previous studies have found overexpression of PDE5 in breast [22] and colon [23] cancers compared to normal tissue, and knock-down of PDE5 expression in these cells resulted in apoptosis [24, 25]. Furthermore, PDE5 inhibition by the non-steroidal anti-inflammatory drug Sulindac and its derivatives caused apoptosis in breast [26], bladder, [27], lung [28] and colon [29-32] cancer. Collectively, these data suggest that a 
persistent suppression of the cGMP pathway by an active PDE5 provides survival advantage to cancer cells, while PDE5 inhibition offers an excellent therapeutic target.

Recent phase II clinical trials in HNSCC patients found that Tadalafil enhanced anti-tumor immunity by inhibiting myeloid derived suppressor cells and increasing tumor infiltrating cytotoxic T-cells $[33,34]$. These trials were based on a previous report that PDE5 inhibitors reduced colon and mammary tumor growth in mice via antitumor immunity [35]. Our results unequivocally demonstrated direct apoptotic effects of Tadalafil on HNSCC cells in vitro. Because the $n u / n u$ mice used in our study are not completely immune-compromised [36, 37], it is conceivable that the direct suppressive effects of Tadalafil on tumor growth in vivo were augmented by an activation of immune mechanisms. Consequently, a dual action of PDE5 inhibitors: as direct suppressors of HNSCC growth and as immune modulators, is of significant therapeutic merit.

Tadalafil was administered to mice by osmotic mini pumps, which deliver a constant low dose of the drug. In humans, Tadalafil is provided in tablet form, at doses ranging from $2.5 \mathrm{mg}$ to $40 \mathrm{mg}$ per day. Compared to other approved PDE5 inhibitors, Tadalafil has a longer serum half-life (17.5 h vs. $4 \mathrm{~h}$ for Sildenafil and Vardenafil) [38]. Although its serum concentration peaks within $2 \mathrm{~h}$ of an oral dose, once daily administration of Tadalafil leads to steady state concentrations after five days [39]. Future studies should consider whether a daily Tadalafil administration or another dosing strategy would be most effective for tumor suppression.

The major downstream targets of cGMP are two serine-threonine kinases, PKG-I and PKG-II. The four HNSCC cell lines expressed PKG-I $\beta$ and PGK-II, while none expressed detectable levels of PKG-I $\alpha$. The PKG isozymes have highly homologous catalytic domains, but differ in their N-terminal domains, which determine their subcellular localization and substrate specificity 
[40]. PKG-I $\alpha$ was reported have pro-tumorigenic effects in lung [41], and ovarian cancer [42], while overexpression of PKG-I $\beta$ was antineoplastic in breast [43] and colon cancer [44]. In addition, overexpression of PKG-II reduced colony formation and proliferation in glioma cells [45]. It is likely that cell-specific differential expression of the PKG isozymes can lead to divergent effects of cGMP elevating drugs on cell survival.

In summary, our studies show that HNSCC cells express critical components of the sGC/PDE/PKG signaling axis, and respond to sGC stimulators and PDE5 inhibitors with increased cGMP, reduced cell viability and apoptosis. Several FDA-approved drugs for treating non-malignant conditions act by increasing cGMP, either by activating sGC or inhibiting PDEs. These drugs can be repurposed as novel chemotherapeutics in head and neck cancer.

\section{Acknowledgements}

This investigation was funded by NIH grants CA096613 and ES020909, DOD grants AR110050 and BC122992, and a pilot grant from Marlene Harris-Ride Cincinnati. We thank Drs William Barrett, of the Department of Radiation Oncology, and Keith Casper, of the Department of Otolaryngology, for providing financial support for these studies. 


\section{References}

[1] C.R. Leemans, B.J.M. Braakhuis, R.H. Brakenhoff, The molecular biology of head and neck cancer, Nat Rev Cancer, 11 (2011) 9-22.

[2] N. Denaro, E.G. Russi, V. Adamo, M.C. Merlano, State-of-the-art and emerging treatment options in the management of head and neck cancer: news from 2013, Oncology, 86 (2014) 212229.

[3] L. Kim, T. King, M. Agulnik, Head and neck cancer: changing epidemiology and public health implications, Oncology (Williston Park), 24 (2010) 915-919, 924.

[4] C. Fung, J.R. Grandis, Emerging drugs to treat squamous cell carcinomas of the head and neck, Expert Opin Emerg Drugs, 15 (2010) 355-373.

[5] F. Fallahian, F. Karami-Tehrani, S. Salami, M. Aghaei, Cyclic GMP induced apoptosis via protein kinase $\mathrm{G}$ in oestrogen receptor-positive and -negative breast cancer cell lines, Febs J, 278 (2011) 3360-3369.

[6] A.M. Fajardo, G.A. Piazza, H.N. Tinsley, The role of cyclic nucleotide signaling pathways in cancer: targets for prevention and treatment, Cancers (Basel), 6 (2014) 436-458.

[7] K. Mujoo, V.G. Sharin, E. Martin, B.-K. Choi, C. Sloan, L.E. Nikonoff, et al., Role of soluble guanylyl cyclase-cyclic GMP signaling in tumor cell proliferation, Nitric Oxide, 22 (2010) 4350 .

[8] D.D. Browning, I.-K. Kwon, R. Wang, cGMP-dependent protein kinases as potential targets for colon cancer prevention and treatment, Future Med Chem, 2 (2010) 65-80.

[9] M. Kumazoe, K. Sugihara, S. Tsukamoto, Y. Huang, Y. Tsurudome, T. Suzuki, et al., 67-kDa laminin receptor increases cGMP to induce cancer-selective apoptosis, J Clin Invest, 123 (2013) 787-799.

[10] H.-C. Wen, C.-P. Chuu, C.-Y. Chen, S.-G. Shiah, H.-J. Kung, K.-L. King, et al., Elevation of soluble guanylate cyclase suppresses proliferation and survival of human breast cancer cells, PLoS One, 10 (2015) e0125518.

[11] E.R. Derbyshire, M.A. Marletta, Structure and regulation of soluble guanylate cyclase, Annu Rev Biochem, 81 (2012) 533-559.

[12] O. Gileadi, Structures of soluble guanylate cyclase: implications for regulatory mechanisms and drug development, Biochem Soc Trans, 42 (2014) 108-113.

[13] M.F. Azevedo, F.R. Faucz, E. Bimpaki, A. Horvath, I. Levy, R.B. de Alexandre, et al., Clinical and molecular genetics of the phosphodiesterases (PDEs), Endocr Rev, 35 (2014) 195233. 
[14] S.H. Francis, J.L. Busch, J.D. Corbin, D. Sibley, cGMP-dependent protein kinases and cGMP phosphodiesterases in nitric oxide and cGMP action, Pharmacol Rev, 62 (2010) 525-563.

[15] P.J. Wright, Comparison of phosphodiesterase type 5 (PDE5) inhibitors, Int J Clin Pract, 60 (2006) 967-975.

[16] D.G. Hottinger, D.S. Beebe, T. Kozhimannil, R.C. Prielipp, K.G. Belani, Sodium nitroprusside in 2014: A clinical concepts review, J Anaesthesiol Clin Pharmacol, 30 (2014) 462471.

[17] J.E. Cannon, J. Pepke-Zaba, Riociguat for pulmonary hypertension, Expert Rev Clin Pharmacol, 7 (2014) 259-270.

[18] E. Bischoff, Potency, selectivity, and consequences of nonselectivity of PDE inhibition, Int J Impot Res, 16 Suppl 1 (2004) S11-14.

[19] N.A.P. Franken, H.M. Rodermond, J. Stap, J. Haveman, C. van Bree, Clonogenic assay of cells in vitro, Nat Protoc, 1 (2006) 2315-2319.

[20] M. Fraser, S.L. Chan, S.S.L. Chan, R.R. Fiscus, B.K. Tsang, Regulation of p53 and suppression of apoptosis by the soluble guanylyl cyclase/cGMP pathway in human ovarian cancer cells, Oncogene, 25 (2006) 2203-2212.

[21] D. Lodygin, A. Menssen, H. Hermeking, Induction of the Cdk inhibitor p21 by LY83583 inhibits tumor cell proliferation in a p53-independent manner, J Clin Invest, 110 (2002) 17171727.

[22] F. Karami-Tehrani, M. Moeinifard, M. Aghaei, M. Atri, Evaluation of PDE5 and PDE9 expression in benign and malignant breast tumors, Arch Med Res, 43 (2012) 470-475.

[23] H.N. Tinsley, B.D. Gary, J. Thaiparambil, N. Li, W. Lu, Y. Li, et al., Colon tumor cell growth-inhibitory activity of sulindac sulfide and other nonsteroidal anti-inflammatory drugs is associated with phosphodiesterase 5 inhibition, Cancer Prev Res (Phila), 3 (2010) 1303-1313.

[24] H.N. Tinsley, B.D. Gary, A.B. Keeton, W. Lu, Y. Li, G.A. Piazza, Inhibition of PDE5 by sulindac sulfide selectively induces apoptosis and attenuates oncogenic Wnt/beta-cateninmediated transcription in human breast tumor cells, Cancer Prev Res (Phila), 4 (2011) 12751284.

[25] B. Zhu, L. Vemavarapu, W.J. Thompson, S.J. Strada, Suppression of cyclic GMP-specific phosphodiesterase 5 promotes apoptosis and inhibits growth in HT29 cells, J Cell Biochem, 94 (2005) 336-350.

[26] H.N. Tinsley, B.D. Gary, A.B. Keeton, W. Zhang, A.H. Abadi, R.C. Reynolds, et al., Sulindac sulfide selectively inhibits growth and induces apoptosis of human breast tumor cells by phosphodiesterase 5 inhibition, elevation of cyclic GMP, and activation of protein kinase G, Mol Cancer Ther, 8 (2009) 3331-3340. 
[27] G.A. Piazza, W.J. Thompson, R. Pamukcu, H.W. Alila, C.M. Whitehead, L. Liu, et al., Exisulind, a novel proapoptotic drug, inhibits rat urinary bladder tumorigenesis, Cancer Res, 61 (2001) 3961-3968.

[28] C.M. Whitehead, K.A. Earle, J. Fetter, S. Xu, T. Hartman, D.C. Chan, et al., Exisulindinduced apoptosis in a non-small cell lung cancer orthotopic lung tumor model augments docetaxel treatment and contributes to increased survival, Mol Cancer Ther, 2 (2003) 479-488.

[29] N. Li, Y. Xi, H.N. Tinsley, E. Gurpinar, B.D. Gary, B. Zhu, et al., Sulindac selectively inhibits colon tumor cell growth by activating the cGMP/PKG pathway to suppress Wnt/betacatenin signaling, Mol Cancer Ther, 12 (2013) 1848-1859.

[30] W.J. Thompson, G.A. Piazza, H. Li, L. Liu, J. Fetter, B. Zhu, et al., Exisulind induction of apoptosis involves guanosine 3',5'-cyclic monophosphate phosphodiesterase inhibition, protein kinase $\mathrm{G}$ activation, and attenuated beta-catenin, Cancer Res, 60 (2000) 3338-3342.

[31] J.D. Whitt, N. Li, H.N. Tinsley, X. Chen, W. Zhang, Y. Li, et al., A novel sulindac derivative that potently suppresses colon tumor cell growth by inhibiting cGMP phosphodiesterase and beta-catenin transcriptional activity, Cancer Prev Res (Phila), 5 (2012) 822-833.

[32] L. Liu, H. Li, T. Underwood, M. Lloyd, M. David, G. Sperl, et al., Cyclic GMP-dependent protein kinase activation and induction by exisulind and CP461 in colon tumor cells, J Pharmacol Exp Ther, 299 (2001) 583-592.

[33] D.T. Weed, J.L. Vella, I.M. Reis, A.C. De la Fuente, C. Gomez, Z. Sargi, et al., Tadalafil reduces myeloid-derived suppressor cells and regulatory $\mathrm{T}$ cells and promotes tumor immunity in patients with head and neck squamous cell carcinoma, Clin Cancer Res, 21 (2015) 39-48.

[34] J.A. Califano, Z. Khan, K.A. Noonan, L. Rudraraju, Z. Zhang, H. Wang, et al., Tadalafil augments tumor specific immunity in patients with head and neck squamous cell carcinoma, Clin Cancer Res, 21 (2015) 30-38.

[35] P. Serafini, K. Meckel, M. Kelso, K. Noonan, J. Califano, W. Koch, et al., Phosphodiesterase-5 inhibition augments endogenous antitumor immunity by reducing myeloidderived suppressor cell function, J Exp Med, 203 (2006) 2691-2702.

[36] S. Ikehara, R.N. Pahwa, G. Fernandes, C.T. Hansen, R.A. Good, Functional T cells in athymic nude mice, Proc Natl Acad Sci U S A, 81 (1984) 886-888.

[37] C.B. Barreto, R.B. Azeredo, R. Fucs, Extrathymic T cells expand in nude mice following different allogeneic stimuli, Immunobiology, 207 (2003) 339-349.

[38] M. Gupta, A. Kovar, B. Meibohm, The clinical pharmacokinetics of phosphodiesterase-5 inhibitors for erectile dysfunction, J Clin Pharmacol, 45 (2005) 987-1003.

[39] S.T. Forgue, B.E. Patterson, A.W. Bedding, C.D. Payne, D.L. Phillips, R.E. Wrishko, et al., Tadalafil pharmacokinetics in healthy subjects, Br J Clin Pharmacol, 61 (2006) 280-288. 
[40] S. Wolfertstetter, J.P. Huettner, J. Schlossmann, cGMP-Dependent Protein Kinase Inhibitors in Health and Disease, Pharmaceuticals (Basel), 6 (2013) 269-286.

[41] J.C. Wong, M. Bathina, R.R. Fiscus, Cyclic GMP/protein kinase G type-Ialpha (PKGIalpha) signaling pathway promotes CREB phosphorylation and maintains higher c-IAP1, livin, survivin, and Mcl-1 expression and the inhibition of PKG-Ialpha kinase activity synergizes with cisplatin in non-small cell lung cancer cells, J Cell Biochem, 113 (2012) 3587-3598.

[42] E.L. Leung, J.C. Wong, M.G. Johlfs, B.K. Tsang, R.R. Fiscus, Protein kinase G type Ialpha activity in human ovarian cancer cells significantly contributes to enhanced Src activation and DNA synthesis/cell proliferation, Mol Cancer Res, 8 (2010) 578-591.

[43] F. Fallahian, F. Karami-Tehrani, S. Salami, Induction of apoptosis by type Ibeta protein kinase $\mathrm{G}$ in the human breast cancer cell lines MCF-7 and MDA-MB-468, Cell Biochem Funct, 30 (2012) 183-190.

[44] Y. Hou, N. Gupta, P. Schoenlein, E. Wong, R. Martindale, V. Ganapathy, et al., An antitumor role for cGMP-dependent protein kinase, Cancer Lett, 240 (2006) 60-68.

[45] F.J. Swartling, M. Ferletta, M. Kastemar, W.A. Weiss, B. Westermark, Cyclic GMPdependent protein kinase II inhibits cell proliferation, Sox9 expression and Akt phosphorylation in human glioma cell lines, Oncogene, 28 (2009) 3121-3131. 


\section{Figure Legends}

Fig 1. $\mathrm{sGC} / \mathrm{cGMP} / \mathrm{PKG}$ signaling pathway in HNSCC cell lines (A) Model of cGMP signaling pathway and the drugs and inhibitors utilized in these studies. Soluble guanylate cyclase (sGC) generates cyclic GMP (cGMP) from GTP, and cGMP is converted to inactive 5' GMP by phosphodiesterase 5 (PDE5). Nitric oxide (NO), generated by sodium nitroprusside (SNP), activates sGC. Direct sGC stimulators (YC-1 or BAY 41-2272) increase sGC activity and synergize with NO. Tadalafil and Sildenafil increase cGMP by inhibiting PDE5. Increased cGMP leads to PKG activation and apoptosis. Inhibitors of sGC (ODQ) or PKG (KT 5823) reverse the apoptotic effects of sGC stimulators and PDE5 inhibitors. (B) Expression of sGC subunits (GUCY1A2, GUCY1A3 and GUCY1B3), PDE5 (PDE5A), PDE11 (PDE11A), PKG-I

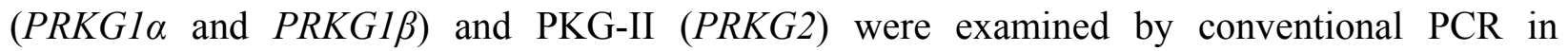
UMSCC-1 (UM1), UMSCC-6 (UM6), UMSCC-47 (UM47) and CAL27 HNSCC cell lines. Human heart cDNA was used as a positive control for all genes except for $P R K G 2$, for which whole human brain cDNA was used. $\beta 2$-microglobulin $(\beta 2 M)$ serves as a control gene. (C) Drug-induced intracellular cGMP accumulation in UM47 and CAL27 cells. Cells were treated with BAY 41-2272 (BAY; $10 \mu \mathrm{M})$, Tadalafil (Tad; $50 \mu \mathrm{M})$, SNP (1mM) or combinations thereof for 60 minutes, and cell lysates were analyzed for cGMP content by competitive ELISA. Results are presented as fold change over vehicle-treated cells. Each value is a mean $\pm \mathrm{SEM}$ of 45 replicates. * indicates significant $(\mathrm{p}<0.05)$ vs. control, \# indicates significant vs BAY, SNP, or Tadalafil alone.

Fig 2. NO donors, sGC stimulators, and PDE5 inhibitors decrease the viability of HNSCC cells. Cells were treated with increasing doses of DETA NONOate, (A) SNAP (B), SNP, (C), BAY 41-2272 (D), YC-1 (E), Tadalafil (F), Sildenafil (G) for 72 h. (H) Cells were treated with 
Tadalafil $(50 \mu \mathrm{M})$ for $2 \mathrm{~h}$ and then with Tadalafil and BAY 41-2272 $(1 \mu \mathrm{M})$ for $72 \mathrm{~h}$. Cell viability was determined by MTT assay. Each value is a mean \pm SEM of 6 replicates. * indicates significant $(\mathrm{p}<0.05)$ vs. control, \# indicates significant vs BAY or Tadalafil alone.

Fig 3. BAY 41-2272 (BAY) and Tadalafil (Tad) decrease cell proliferation and induce apoptosis in HNSCC cells. (A) Cells were treated with BAY $(10 \mu \mathrm{M})$ or Tadalafil $(50 \mu \mathrm{M})$ for $24 \mathrm{~h}$ in the presence of BrdU. After fixation, BrdU incorporation was determined by ELISA. Each value is a mean \pm SEM of four replicates. (B) Cells were treated with BAY $(10 \mu \mathrm{M})$, Tadalafil (50 $\mu \mathrm{M})$ or vehicle (Con) for $72 \mathrm{~h}$, stained with Annexin $\mathrm{V}$ and propidium iodide and analyzed by flow cytometry. Each value is a mean $\pm \mathrm{SEM}$ of three replicates. $*$ indicates significant $(\mathrm{p}<0.05)$ vs. control. (C) CAL27 and UM47 cells were treated with various drugs for $24 \mathrm{~h}$, and cell lysates were analyzed for caspase 9 (Cas-9) by western blot.

Fig 4. BAY 41-2272 (BAY) and Tadalafil (Tad) decrease clonogenic survival of HNSCC cells. CAL27 (A), UM6 (B) and UM47 (C) cells were plated at 150 cells/well in 6 well plates and treated before first doubling. After $72 \mathrm{~h}$, treatments were replaced with growth media and colonies were allowed to form for 2 weeks. Colonies were stained with crystal violet and counted. Quantification is shown in the right panels. Each value is a mean $\pm \mathrm{SEM}$ of three replicates. * indicates significant $(\mathrm{p}<0.05)$ vs. control.

Fig 5. Effect of sGC or PKG inhibitors on apoptosis induced by BAY or Tadalafil. UM47 cells were treated with the PKG inhibitor KT5823 (KT) for $1 \mathrm{~h}$, followed by $72 \mathrm{~h}$ treatment with KT and either BAY $(10 \mu \mathrm{M})$ or Tadalafil $(50 \mu \mathrm{M})$. (A) Cells were stained with Annexin V and propidium iodide $(\mathrm{PI})$ and analyzed by flow cytometry. Each value is a mean $\pm \mathrm{SEM}$ of three replicates. ${ }^{*}$ indicates significant $(\mathrm{p}<0.05)$ vs. control. \# indicates significant vs. BAY or Tadalafil without ODQ or KT. (B) The distribution of live cells (Annexin-/PI-), cells in early 
apoptosis (Annexin $\mathrm{V}+/ \mathrm{PI}-$ ), late apoptosis (Annexin $\mathrm{V}+/ \mathrm{PI}+$ ) and dead/necrotic cells (Annexin(PI+).

Fig 6. Tadalafil suppressed the growth of CAL27 xenografts in vivo. Athymic (nu/nu) mice were inoculated subcutaneously with CAL27 cells. Once tumors reached a volume of $\sim 200 \mathrm{~mm}^{3}$, Alzet osmotic minipumps, delivering Tadalafil $(1 \mathrm{mg} / \mathrm{kg} / \mathrm{day})$ or vehicle were implanted sc. (A) Tumor volumes were measured by caliper. (B) Tumors were excised and weighed 24 days after the start of drug delivery. Each value is a mean \pm SEM of 12 mice. * indicates significant $(p<0.05)$ vs. vehicle treated. (C) Representative photographs of Tadalafil and vehicle treated mice and excised tumors. 

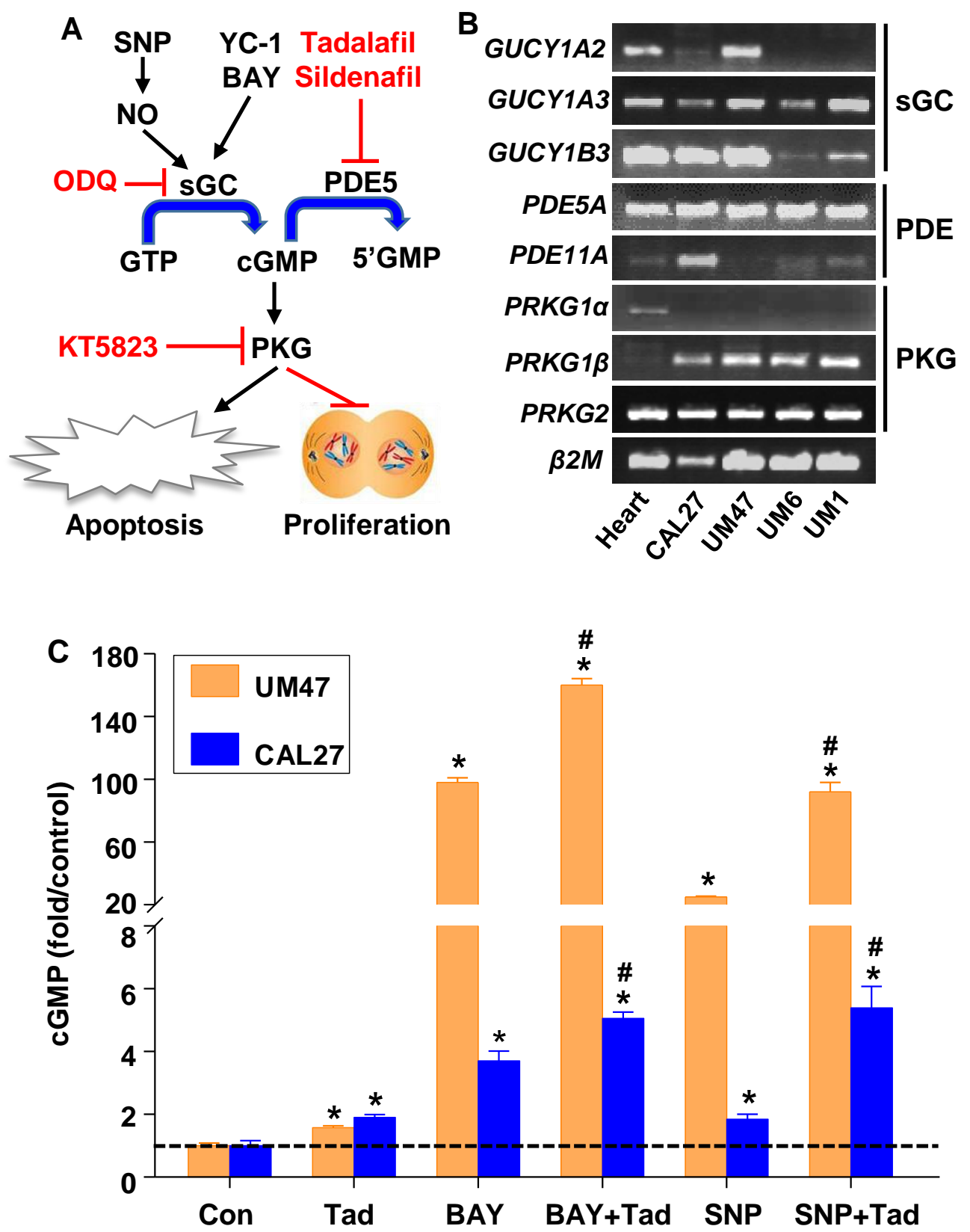

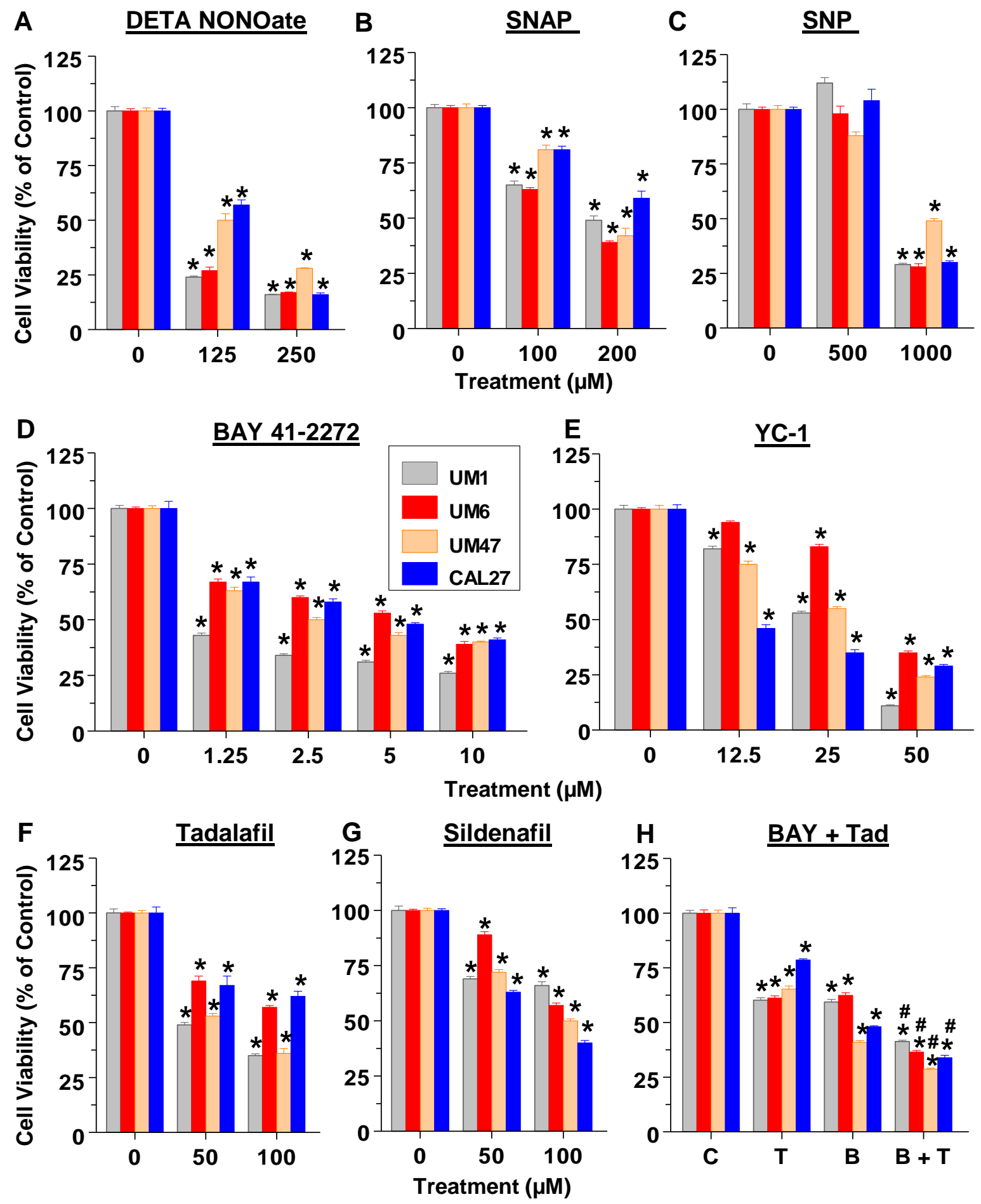
Figure 3
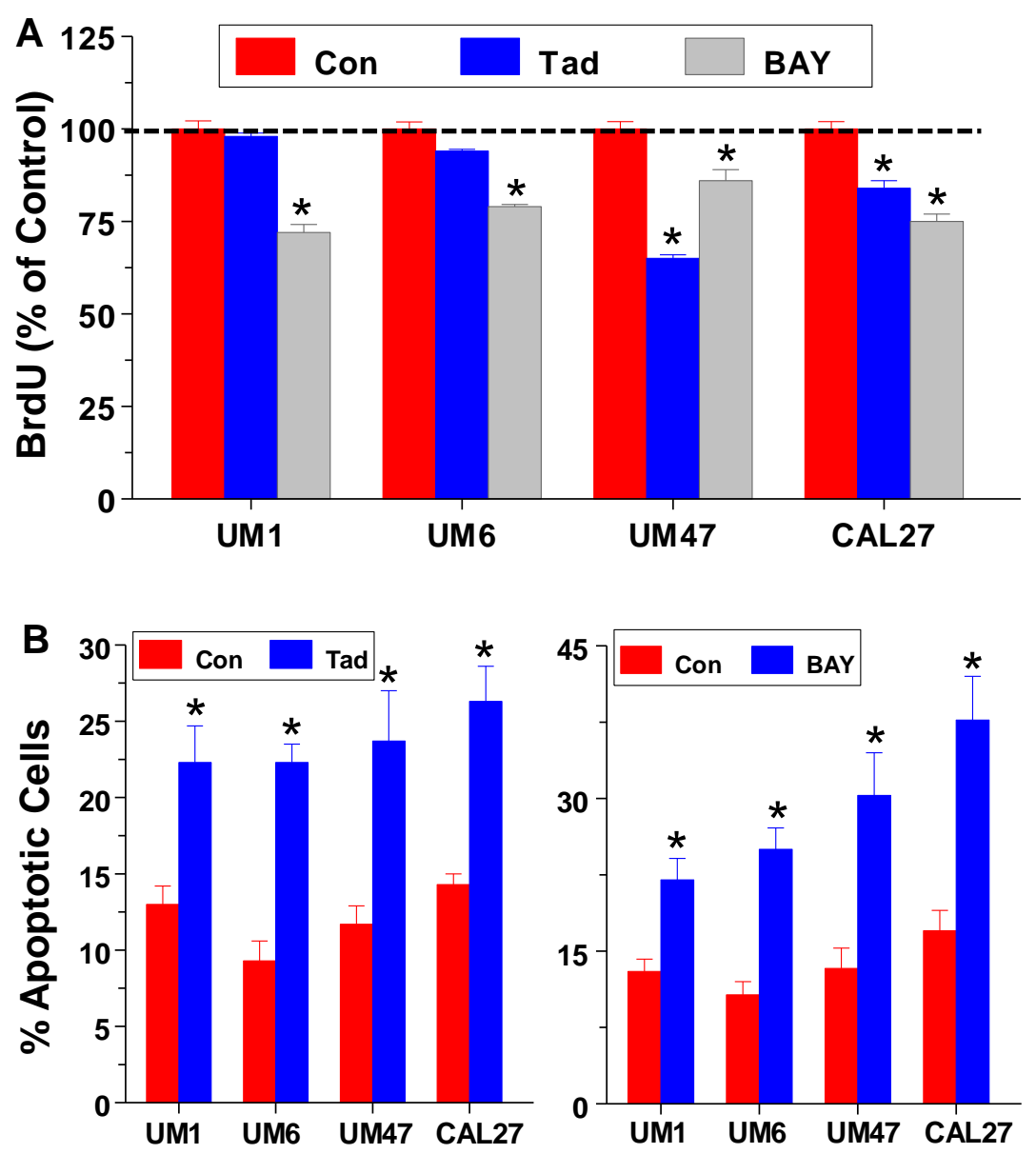

C

CAL27
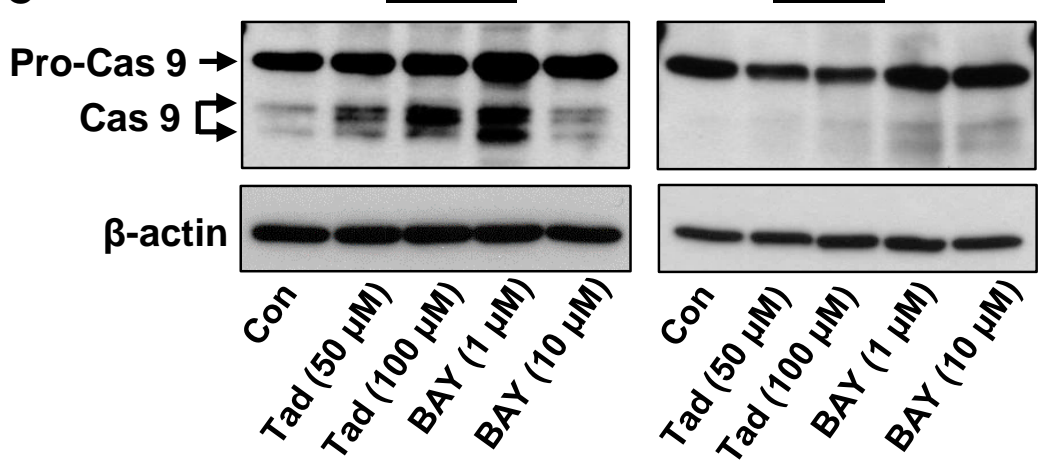

UM47 
Figure 4
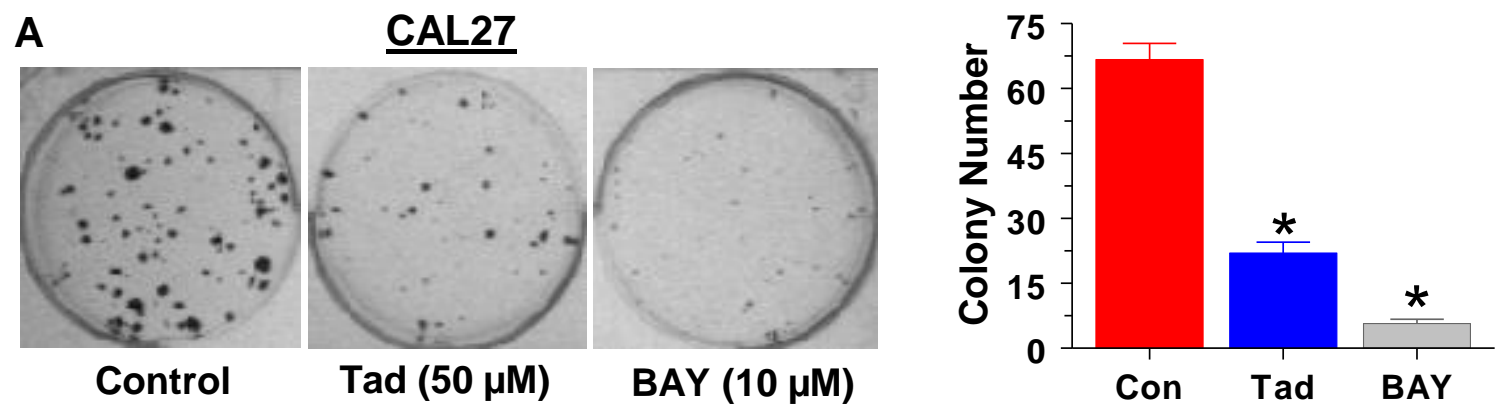

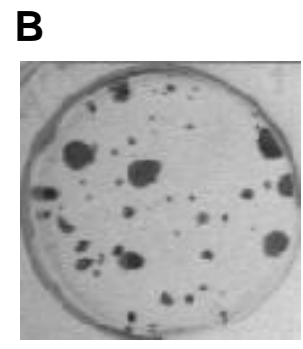

Control

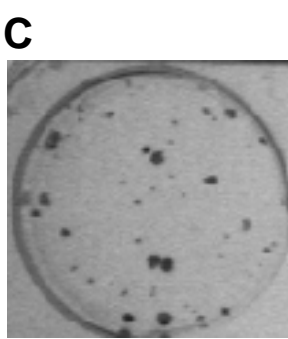

Control
UM6

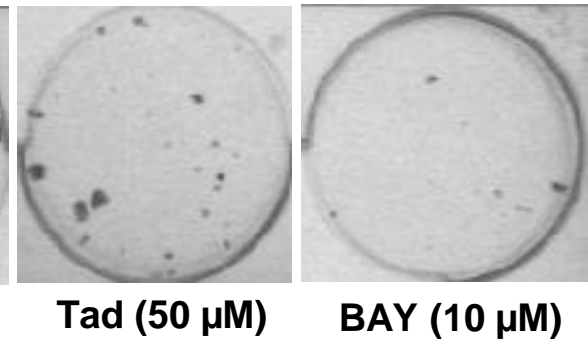

UM47

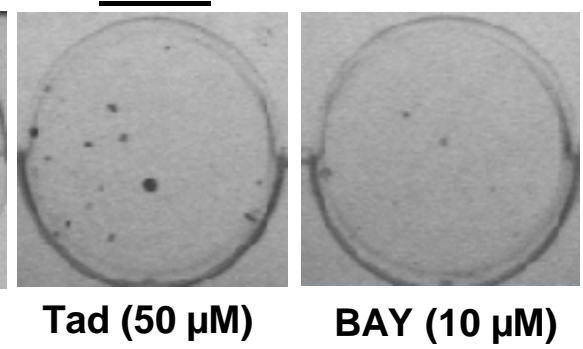

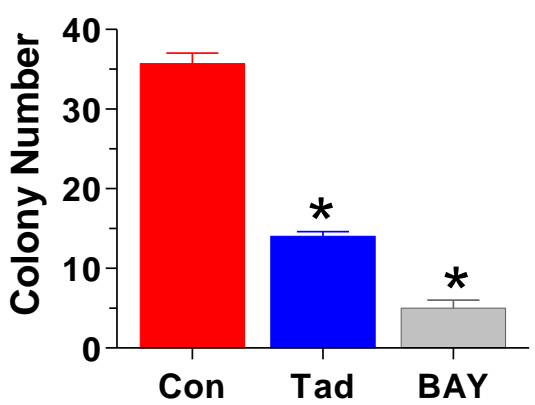

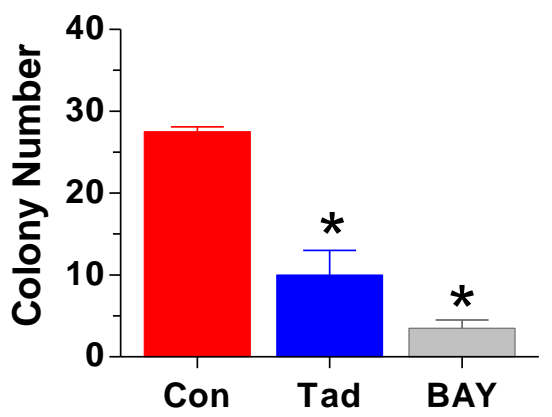




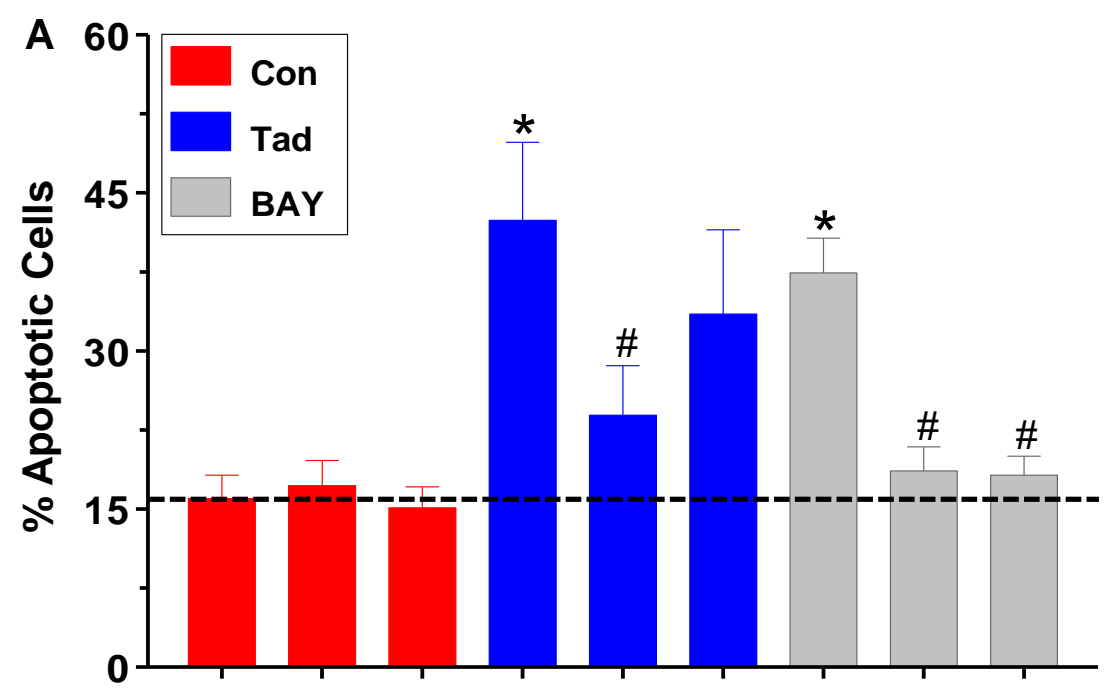

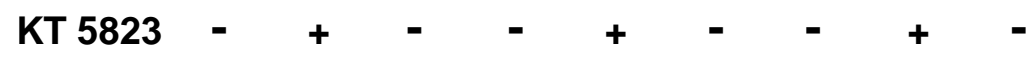

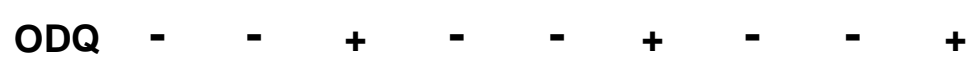

B

\begin{tabular}{|l|c|c|c|c|}
\hline \multicolumn{1}{|c|}{ Treatment } & Live & $\begin{array}{c}\text { Early } \\
\text { Apoptotic }\end{array}$ & $\begin{array}{c}\text { Late } \\
\text { Apoptotic }\end{array}$ & Dead \\
\hline Control & $76.3^{\star}$ & 2.6 & 13.4 & 7.7 \\
\hline KT & 75.3 & 2.7 & 14.4 & 7.5 \\
\hline ODQ & 79.1 & 2.8 & 12.3 & 5.8 \\
\hline Tadalafil & 49.8 & 16.1 & 26.3 & 7.8 \\
\hline Tad + KT & 71.2 & 7.1 & 16.8 & 4.9 \\
\hline Tad + ODQ & 56.8 & 13.1 & 20.3 & 9.7 \\
\hline BAY & 53.3 & 10.7 & 26.7 & 9.3 \\
\hline BAY + KT & 75.1 & 3.8 & 14.8 & 6.3 \\
\hline BAY + ODQ & 74.9 & 4.1 & 14.1 & 6.9 \\
\hline
\end{tabular}

* Percent of total cells 
A

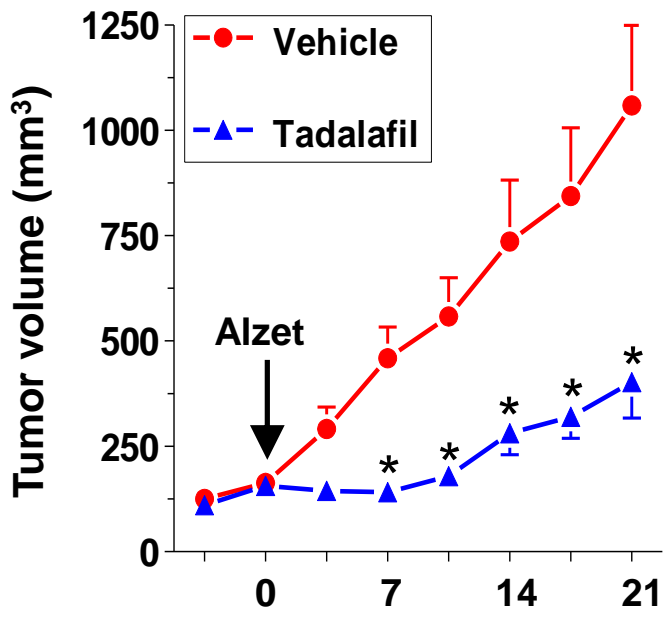

Days of Treatment
B

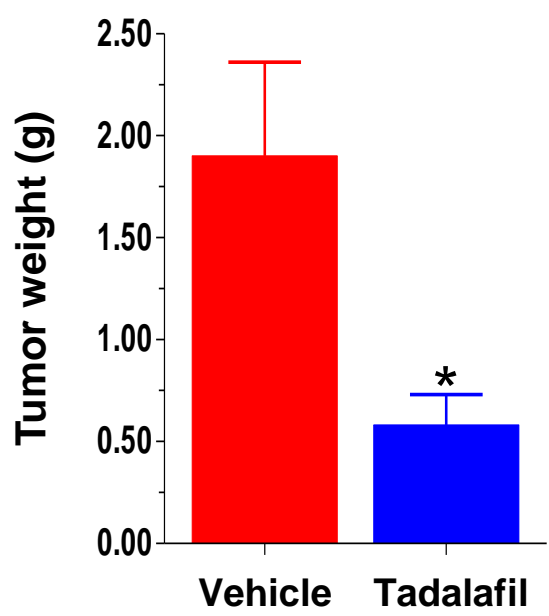

C
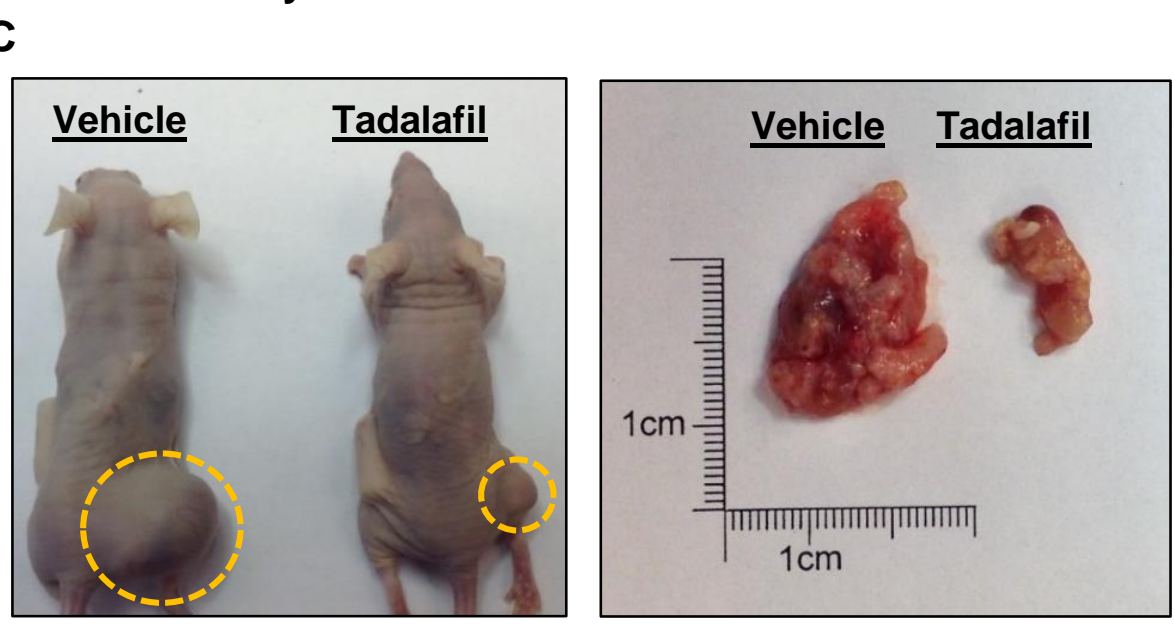
Table I: Primers used in conventional PCR.

\begin{tabular}{ccc}
\hline Gene & \multicolumn{1}{c}{ Forward primer } & \multicolumn{1}{c}{ Reverse primer } \\
\hline GUCY1A2 & AGTGAAACGGATTTGGGTACTGA & TGGACCAGTCCTTACCTCCA \\
\hline GUCY1A3 & ACGAGTGAGGAGATGGGACA & GGTAGAGCCCTCGTCCTGTA \\
\hline GUCY1B3 & ACACACTGCTGGGATACTGC & AGAGCTAGAGGCCAATTATTCCA \\
\hline PDE5A & TTGCCTAGCAGTGAAGTGCC & CAATTGCTTGTGATGGCCTGT \\
\hline PDE11A & CGCGATGTTAACCACTGCTG & GGCAGAGCCACTCTTAGCTT \\
\hline PRKG1a & CGAGTACTTAGCGCCCATTCA & CCGCTTCTCCAGCTCTTTGAT \\
\hline PRKG1ß & TGATCGGAGAGGGGAGGAAG & CGGTACTTGTCCAGCTCGTT \\
\hline PRKG2 & ATCCCAACTCCACCATTTCCT & GTACAATTCTGCATACAGATACCAA \\
\hline B2M & GGCATTCCTGAAGCTGAC & GAATCTTTGGAGTACGCTGG \\
\hline
\end{tabular}

